OPEN ACCESS

Edited by:

Rob McClung,

Dartmouth College, USA

Reviewed by:

Luis Vidali,

Worcester Polytechnic Institute, USA Halina Gabrys,

Jagiellonian University, Poland

${ }^{*}$ Correspondence:

Masamitsu Wada

masamitsu.wada@gmail.com

Specialty section:

This article was submitted to

Plant Cell Biology,

a section of the journal

Frontiers in Plant Science

Received: 20 November 2015

Accepted: 11 April 2016

Published: 03 May 2016

Citation:

SuetsuguN and Wada M (2016)

Evolution of the Cp-Actin-based Motility System of Chloroplasts

in Green Plants.

Front. Plant Sci. 7:561.

doi: 10.3389/fpls.2016.00561

\section{Evolution of the Cp-Actin-based Motility System of Chloroplasts in Green Plants}

\author{
Noriyuki Suetsugu ${ }^{1}$ and Masamitsu Wada ${ }^{2 *}$ \\ 1 Graduate School of Biostudies, Kyoto University, Kyoto, Japan, ${ }^{2}$ Department of Biological Sciences, Graduate School of \\ Science and Engineering, Tokyo Metropolitan University, Tokyo, Japan
}

During the course of green plant evolution, numerous light responses have arisen that optimize their growth under fluctuating light conditions. The blue light receptor phototropin mediates several photomovement responses at the tissue, cellular and organelle levels. Chloroplast photorelocation movement is one such photomovement response, and is found not only in most green plants, but also in some red algae and photosynthetic stramenopiles. In general, chloroplasts move toward weak light to maximally capture photosynthetically active radiation (the chloroplast accumulation response), and they move away from strong light to avoid photodamage (the avoidance response). In land plants, chloroplast movement is dependent on specialized actin filaments, chloroplast-actin filaments (cp-actin filaments). Through molecular genetic analysis using Arabidopsis thaliana, many molecular factors that regulate chloroplast photorelocation were identified. In this Perspective, we discuss the evolutionary history of the molecular mechanism for chloroplast photorelocation movement in green plants in view of cp-actin filaments.

Keywords: blue light, chloroplast, cp-actin filament, green plants, phototropin

\section{INTRODUCTION}

Green plants (land plants and green algae) have made many evolutionary innovations, moving from aquatic to terrestrial habitats, with multiple evolutions of multicellularity, and complex multicellular development. These evolutionary innovations also include numerous photomovement responses at the tissue, cell, and organelle levels, which allow organisms to adapt to fluctuating ambient light conditions. Chloroplast photorelocation movement (hereafter, chloroplast movement) is found in a broad range of plant and algal species including chlorophyte and charophyte green algae and land plants (Viridiplantae: Chlorophyta and Streptophyta), red algae (Rhodophyta), and photosynthetic stramenopiles (Senn, 1908). It has been extensively studied particularly in Streptophyta: embryophytes (land plants, including bryophytes, lycophytes, ferns, and seed plants) as well as Zygnemataceae (including Mougeotia and Mesotaenium) and Klebsormidiophyceae (such as Klebsormidium, formerly named Hormidium; Senn, 1908; for review, see Haupt and Scheuerlein, 1990) (Figure 1). Blue light is the most effective means of inducing chloroplast movement though red light is also effective in some ferns, mosses, and green algae (for review, see Suetsugu and Wada, 2007).

In most land plants, where cells have many small chloroplasts, the chloroplasts move toward weak light to capture light efficiently (the accumulation response), and they move away from 
strong light to reduce photodamage (the avoidance response). In general, these chloroplast movements are dependent on actin filaments in most plant species with some exceptions. In some bryopsis algae (Chlorophyta: Ulvophyceae), such as Dichotomosiphon and Bryopsis, most chloroplasts are detached from the plasma membrane and so blue light-induced chloroplast accumulation is mediated by the slowing of microtubuledependent cytoplasmic streaming at the irradiated site, rather than by autonomous directional movement of individual chloroplasts (Mizukami and Wada, 1981; Maekawa et al., 1986). Members of the filamentous green algae Mougeotia and Klebsormidium, as well as the single-celled Mesotaenium, each has a single large chloroplast in a cylindrical cell. Klebsormidium exhibits light-induced chloroplast movement along the plasma membrane (Senn, 1908; for review, see Haupt and Scheuerlein, 1990). In Mougeotia and Mesotaenium, the ribbon-shaped chloroplast is sandwiched between vacuoles, and only the edge of the chloroplast attaches to the plasma membrane. In these algae chloroplast movement is due to the rotation of the chloroplast around the central axis of the cell (Senn, 1908; for review, see Haupt and Scheuerlein, 1990), using actin filaments (Wagner et al., 1972; Mineyuki et al., 1995). In streptophytes, microtubule-dependent chloroplast movement has only been found in the moss Physcomitrella patens, and this moss can use both microtubules and actin filaments for chloroplast movement (Sato et al., 2001). Thus, it is plausible that actin-dependent chloroplast photorelocation movement along the plasma membrane may have arisen subsequent to the divergence of Chlorophyta and Streptophyta (Figure 1) and $P$. patens evolved a microtubule-dependent system independently during land plant evolution. While algae can generally control light capture by movement in water, land plants are sessile and thus must adapt to fluctuating light conditions, such as shading by and sudden sunbeams streaming through the neighboring plants. To adapt to the harsh light conditions on land, land plants have evolved a motility system for chloroplast movement and positioning, using specialized short actin filaments around the chloroplasts, chloroplast-actin filaments (cp-actin filaments; Kadota et al., 2009; Yamashita et al., 2011; Tsuboi and Wada, 2012; Kong et al., 2013). These filaments are generated (polymerized) and employed for movement at the interface between the chloroplast and the plasma membrane (Kadota et al., 2009; Kong et al., 2013). In this Perspective, we discuss the evolution of actin-dependent chloroplast movement, and its molecular components, in green plants.

\section{MOLECULAR FACTORS REGULATING CHLOROPLAST MOVEMENT IDENTIFIED IN Arabidopsis thaliana}

Through the analysis of Arabidopsis thaliana mutants that are defective in chloroplast movement, many components of the photorelocation system have been identified (for review, see Wada and Suetsugu, 2013) (Figure 2). These include the photoreceptor kinase phototropin (phot; Jarillo et al., 2001; Kagawa et al., 2001; Sakai et al., 2001); an actin-binding protein CHLOROPLAST UNUSUAL POSITIONING 1 (CHUP1; Oikawa et al., 2003); the kinesin-like protein KINESIN-LIKE PROTEIN FOR ACTIN-BASED CHLOROPLAST MOVEMENT (KAC; Suetsugu et al., 2010); a C2 domain protein PLASTID

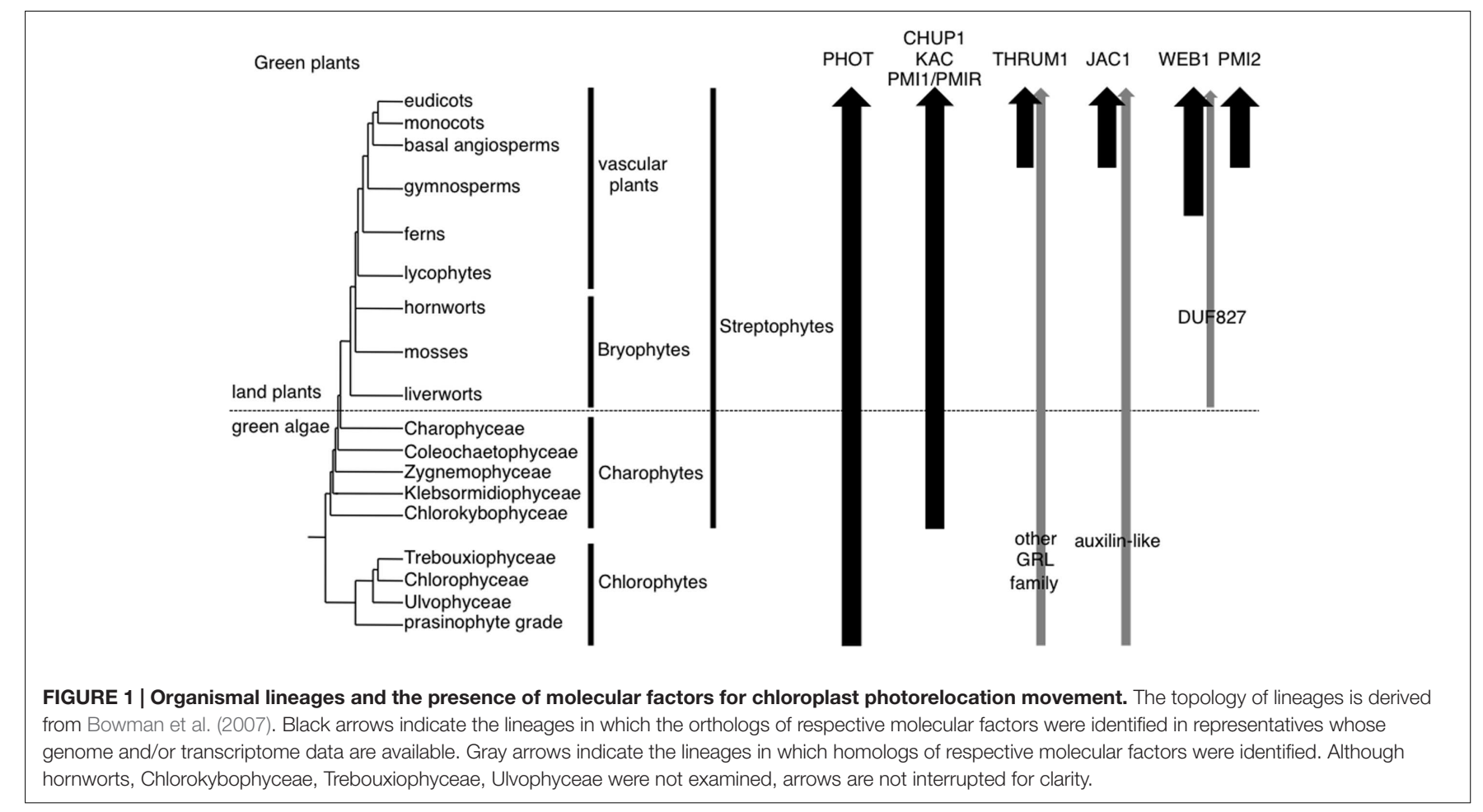




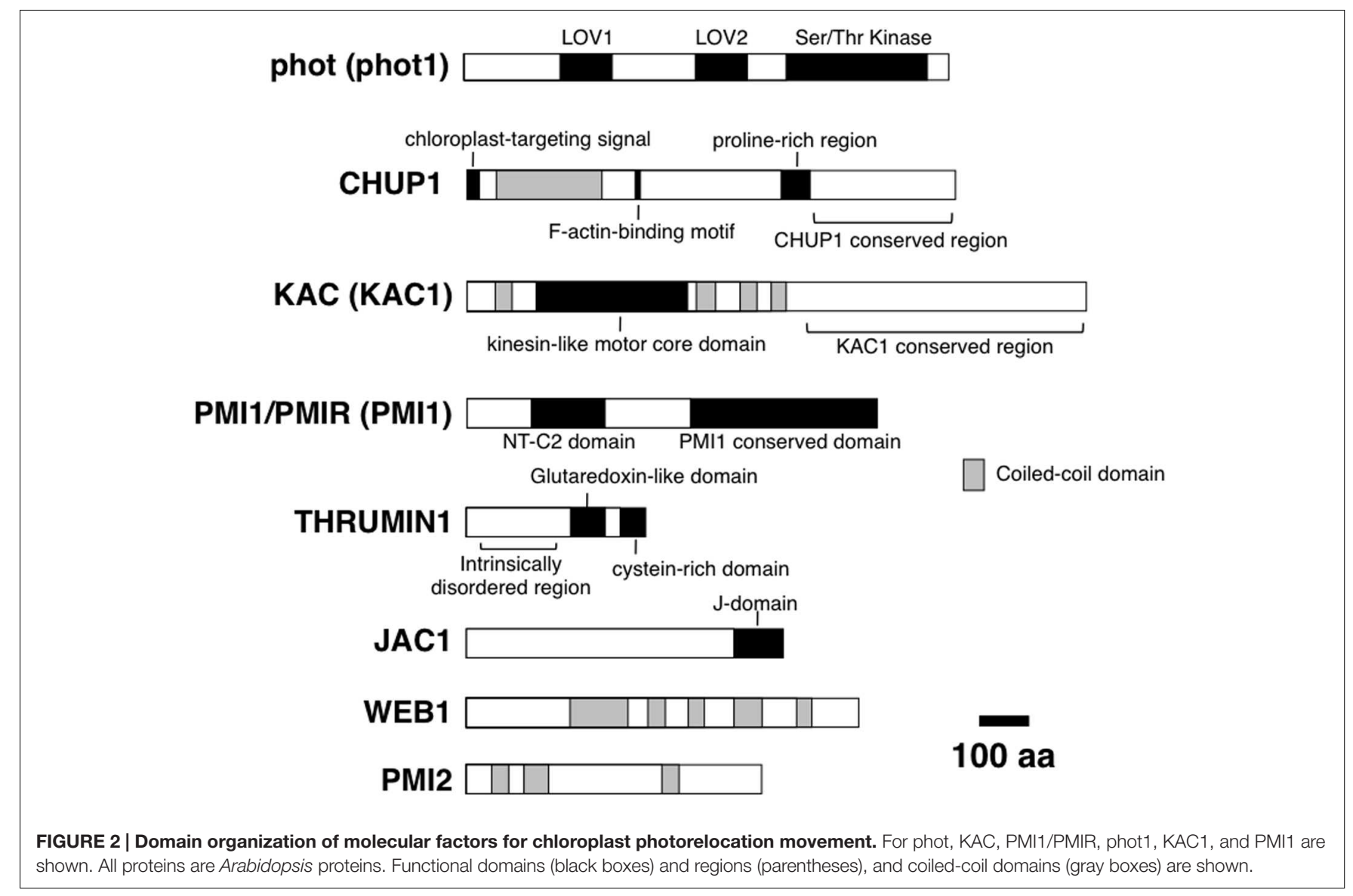

MOVEMENT IMPAIRED 1 (PMI1; DeBlasio et al., 2005); a glutaredoxin-like protein THRUMIN 1 (THRUM1; Whippo et al., 2011); an auxilin-like protein J-DOMAIN PROTEIN REQUIRED FOR CHLOROPLAST ACCUMULATION RESPONSE 1 (JAC1; Suetsugu et al., 2005); two related coiledcoil proteins WEB1 (WEAK CHLOROPLAST MOVEMENT UNDER BLUE LIGHT 1) and PMI2 (PLASTID MOVEMENT IMPAIRED 2; Luesse et al., 2006; Kodama et al., 2010). We have demonstrated that these eight proteins (and their homologs) are essential for chloroplast movement and positioning via $\mathrm{cp}$-actin filaments. Phototropins mediate chloroplast photorelocation movement via blue-light-dependent generation of cp-actin filaments (Kadota et al., 2009; Ichikawa et al., 2011; Kong et al., 2013). CHUP1 and KAC are essential for the generation of cp-actin filaments (Kadota et al., 2009; Suetsugu et al., 2010; Kong et al., 2013) and the actin-binding activity has been shown in vitro (Oikawa et al., 2003; Schmidt von Braun and Schleiff, 2008; Suetsugu et al., 2010). Both chup1 and kac (kaclkac2 double) mutants in A. thaliana are defective in chloroplast photorelocation movement and the attachment of chloroplasts to the plasma membrane (Oikawa et al., 2003, 2008; Suetsugu et al., 2010). THRUM1 is required for the efficient generation of cp-actin filaments and chloroplast movement, and co-localized with cp-actin filaments in vivo (Kong et al., 2013). PMI1 is required for stability of cp-actin filaments (Suetsugu et al., 2015). JAC1, WEB1, and PMI2 are involved in blue-light-induced reorganization of cp-actin filaments, although they are not essential to the generation of cp-actin filaments (Kodama et al., 2010; Ichikawa et al., 2011). These factors can be classified roughly into three categories: photoreceptor (phot), motility (CHUP1, KAC, THRUM1, PMI1), and signal transduction (JAC1, WEB1, and PMI2). This classification is based on the phenotypes in cp-actin filaments in respective mutant plants; cp-actin filaments were severely reduced or not detected in mutants deficient in factors classified as a motility category, whereas the amount of cp-actin filaments was not changed but the light-regulation was impaired in mutants deficient in factors classified as a signal transduction category. Because chloroplast movement is found universally in green algae and land plants, we subsequently investigated whether the molecular factors identified in A. thaliana are conserved across green algae and land plants.

\section{PHOTORECEPTORS AND THE REGULATOR FOR MOTILITY SYSTEM ARE CONSERVED IN STREPTOPHYTA}

The molecular factors for chloroplast photorelocation movement in green plant species other than $A$. thaliana, the fern Adiantum capillus-veneris, and $P$. patens, were not reported 
previously. As a comprehensive analysis of phototropin gene phylogeny has been already published (Li et al., 2014, 2015), we focused our search on the orthologs of CHUP1, KAC, PMI1, THRUM1, JAC1, WEB1, and PMI2. In addition to searching in NCBI GenBank's $\mathrm{nr}$ database and the JGI genome database, we used the published transcriptome data from the fern Lygodium japonicum (Aya et al., 2015) and five charophyte algae (Spirogyra pratensis, Nitella mirabilis, Coleochaete orbicularis, Klebsormidium flaccidum, and Mesostigma viride; Ju et al., 2015). No orthologous proteins of these factors, including phototropins, were identified in sequence data from non-green plants, i.e., orthologs were only found in green plants. Chlorophytes do not have orthologous proteins of CHUP1, KAC, PMI1, THRUM1, JAC1, WEB1, or PMI2, but have a phototropin (Li et al., 2015). We found that there are CHUP1, KAC, and PMI1 orthologous proteins in charophyte algae as well as land plants (Figure 1). It was shown that CHUP1, and KAC orthologs mediated chloroplast photorelocation movement and the attachment of chloroplasts to the plasma membrane in the fern $A$. capillus-veneris and the moss $P$. patens (Suetsugu et al., 2012; Usami et al., 2012; Shen et al., 2015). In each case, cp-actin filaments were detected (Yamashita et al., 2011; Tsuboi and Wada, 2012). Furthermore, although $P$. patens uses both actin filaments and microtubules for chloroplast movements (Sato et al., 2001), CHUP1 and KAC orthologs specifically mediated the actin-dependent movements (Usami et al., 2012; Shen et al., 2015). These results suggest that the motility system using cp-actin filaments is likely to be conserved throughout land plants. A. thaliana has two PMI1-related proteins, PMIR1 and PMIR2: PMIR1 mediates blue light-induced plastid movement via cp-actin filaments in epidermal cells, together with PMI1 (Suetsugu et al., 2015). PMI1 and PMIR are likely to have diverged before the gymnosperm and angiosperm divergence (Suetsugu et al., 2015), suggesting that PMI1/PMIR homologs in charophyte algae and non-seed plants should be able to regulate cp-actin-filament-mediated chloroplast movement.

THRUM1 belongs to the glutaredoxin-like (GRL) protein family (of which there are 15 in A. thaliana); rice has the THRUM1-orthologous proteins (Navrot et al., 2006; Whippo et al., 2011). JAC1 belongs to the clathrin-uncoating factor auxilin-like proteins (of which there are seven in A. thaliana); rice and probably other monocot species have JAC1-orthologous proteins (Suetsugu et al., 2005). However, it remained to be determined whether these monocot THRUM1-like or JAC1like genes are also involved in chloroplast movement. WEB1 and PMI2 belong to the DUF827 coiled-coil protein family which is divided into four subfamilies, WEB1, PMI2, WPRa, and WPRb; conifers have WEB1-orthologous proteins (Kodama et al., 2011). Although all streptophytes studied to date have GRL proteins and auxilin-like proteins (DUF827 proteins are found only in land plants), we could not identify direct orthologs of THRUM1, JAC1, WEB1, or PMI2 in charophyte algae or non-seed plants (Figure 1). Compared with vascular plants, the liverwort Marchantia polymorpha and the moss
P. patens require much higher fluence rates of blue light to induce the avoidance response (Kadota et al., 2000; Sato et al., 2001; Komatsu et al., 2014). Our genetic analyses in A. thaliana suggest that WEB1 and PMI2 are necessary for suppression of the accumulation response (probably the suppression of JAC1 activity) under strong blue light (Kodama et al., 2010). Strong blue light can activate the signal transduction pathway for both the accumulation and avoidance responses (for review, see Suetsugu and Wada, 2007). Thus, the suppression of the accumulation response should be required for an efficient induction of the avoidance response, under strong light conditions. Thus, the evolution of JAC1, WEB1, and PMI2 may have permitted a more efficient avoidance response in land plants.

Thus, phot, CHUP1, KAC, and PMI1/PMIR proteins are core factors for chloroplast movement in Streptophytes. In A. thaliana, mutants deficient in phot, CHUP1, KAC, or PMI1/PMIR proteins exhibit severe defects in chloroplast movement and positioning (Sakai et al., 2001; Oikawa et al., 2003, 2008; Suetsugu et al., 2010, 2015) whereas mutants deficient in THRUM1, JAC1, WEB1, and PMI2 show only partial defects in chloroplast movement (Suetsugu et al., 2005; Luesse et al., 2006; Kodama et al., 2010; Whippo et al., 2011).

Although cp-actin filaments were found in A. thaliana, A. capillus-veneris, and $P$. patens, different structures of actin filaments associated with chloroplast movements were observed in some plant species using different experimental procedures (Kadota and Wada, 1989; Kandasamy and Meagher, 1999; Kumatani et al., 2006; Anielska-Mazur et al., 2009). Thus, we need to observe dynamics of actin filaments in various species using the same procedure in which cp-actin filaments were examined in A. thaliana. Nevertheless, the conservation of CHUP1 and KAC in streptophytes suggests that chloroplast movement in charophyte algae such as Klebsormidium, Mougeotia, and Mesotaenium might be regulated by cp-actin filaments. In Mougeotia cells fixed after irradiation with strong white light, short actin filaments have been observed at the leading edge of the moving chloroplast (Mineyuki et al., 1995), implying the presence of cp-actin filaments in Mougeotia. Further exploration of genetic model systems in charophyte algae will be required to elucidate the conservation across Streptophyta of the motility system for chloroplast photorelocation movement.

\section{AUTHOR CONTRIBUTIONS}

All authors listed, have made substantial, direct and intellectual contribution to the work, and approved it for publication.

\section{ACKNOWLEDGMENT}

This work was supported by the Grant-in-Aid for Scientific Research to NS (no. 26840097) and to MW (no. 20227001, 23120523, 25120721, 25251033) from the Japan Society for the Promotion of Science. 


\section{REFERENCES}

Anielska-Mazur, A., Bernaś, T., and Gabryś, H. (2009). In vivo reorganization of the actin cytoskeleton in leaves of Nicotiana tabacum L. transformed with plastinGFP. Correlation with light-activated chloroplast responses. BMC Plant Biol. 9:64. doi: 10.1186/1471-2229-9-64

Aya, K., Kobayashi, M., Tanaka, J., Ohyanagi, H., Suzuki, T., Yano, K., et al. (2015). De novo transcriptome assembly of a fern, Lygodium japonicum, and a web resource database, Ljtrans DB. Plant Cell Physiol. 56:e5. doi: $10.1093 / \mathrm{pcp} / \mathrm{pcu} 184$

Bowman, J. L., Floyd, S. K., and Sakakibara, K. (2007). Green genesComparative genomics of the green branch of life. Cell 129, 229-234. doi: 10.1016/j.cell.2007.04.004

DeBlasio, S. L., Luesse, D. L., and Hangarter, R. P. (2005). A plant-specific protein essential for blue-light-induced chloroplast movements. Plant Physiol. 139, 101-114. doi: 10.1104/pp.105.061887

Haupt, W., and Scheuerlein, R. (1990). Chloroplast movement. Plant Cell Environ. 13, 595-614. doi: 10.1111/j.1365-3040.1990. tb01078.x

Ichikawa, S., Yamada, N., Suetsugu, N., Wada, M., and Kadota, A. (2011). Red light, phot1, and JAC1 modulate phot2-dependent reorganization of chloroplast actin filaments and chloroplast avoidance movement. Plant Cell Physiol. 52, 1422-1432. doi: 10.1093/pcp/pcr087

Jarillo, J. A., Gabrys, H., Capel, J., Alonso, J. M., Ecker, J. R., and Cashmore, A. R. (2001). Phototropin-related NPL1 controls chloroplast relocation induced by blue light. Nature 410, 952-954. doi: 10.1038/ 35073622

Ju, C., Van de Poel, B., Cooper, E. D., Thierer, J. H., Gibbons, T. R., Delwiche, C. F., et al. (2015). Conservation of ethylene as a plant hormone over 450 million years of evolution. Nat. Plant 1:14004. doi: 10.1038/NPLANT. 2014.4

Kadota, A., Sato, Y., and Wada, M. (2000). Intracellular chloroplast photorelocation in the moss Physcomitrella patens is mediated by phytochrome as well as by a blue-light receptor. Planta 210, 932-937. doi: $10.1007 / \mathrm{s} 004250050700$

Kadota, A., and Wada, M. (1989). Photoinduction of circular F-actin on chloroplast in a fern protonemal cell. Protoplasma 151, 171-174. doi: 10.1007/BF01403456

Kadota, A., Yamada, N., Suetsugu, N., Hirose, M., Saito, C., Shoda, K., et al. (2009). Short actin-based mechanism for light-directed chloroplast movement in Arabidopsis. Proc. Natl. Acad. Sci. U.S.A. 106, 13106-13111. doi: 10.1073/pnas.0906250106

Kagawa, T., Sakai, T., Suetsugu, N., Oikawa, K., Ishiguro, S., Kato, T., et al. (2001). Arabidopsis NPL1: a phototropin homolog controlling the chloroplast high-light avoidance response. Science 291, 2138-2141. doi: 10.1126/science.291.5511.2138

Kandasamy, M., and Meagher, M. (1999). Actin-organelle interaction: Association with chloroplast in Arabidopsis leaf mesophyll cells. Cell Motil. Cytoskeleton 44, 110-118. doi: 10.1002/(SICI)1097-0169(199910)44

Kodama, Y., Suetsugu, N., Kong, S.-G., and Wada, M. (2010). Two interacting coiled-coil proteins, WEB1 and PMI2, maintain the chloroplast photorelocation movement velocity in Arabidopsis. Proc. Natl. Acad. Sci. U.S.A. 107, 1959119596. doi: 10.1073/pnas.1007836107

Kodama, Y., Suetsugu, N., and Wada, M. (2011). Novel protein-protein interaction family proteins involved in chloroplast movement response. Plant Signal. Behav. 6, 483-490. doi: 10.4161/psb.6.4.14784

Komatsu, A., Terai, M., Ishizaki, K., Suetsugu, N., Tsuboi, H., Nishihama, R., et al. (2014). Phototropin encoded by a single-copy gene mediates chloroplast photorelocation movements in the liverwort Marchantia polymorpha. Plant Physiol. 166, 411-427. doi: 10.1104/pp.114.245100

Kong, S.-G., Arai, Y., Suetsugu, N., Yanagida, T., and Wada, M. (2013). Rapid severing and motility of chloroplast-actin filaments are required for the chloroplast avoidance response in Arabidopsis. Plant Cell 25, 572-590. doi: 10.1105/tpc.113.109694

Kumatani, T., Sakurai-Ozato, N., Miyawaki, N., Yokota, E., Shimmen, T., and Takagi, S. (2006). Possible association of actin filaments with chloroplasts of spinach mesophyll cells in vivo and in vitro. Protoplasma 229, 45-52. doi: 10.1007/s00709-006-0189-8
Li, F.-W., Rothfels, C. J., Melkonian, M., Villarreal, J. C., Stevenson, D. W., Graham, S. W., et al. (2015). The origin and evolution of phototropins. Front. Plant Sci. 6:637. doi: 10.3389/fpls.2015.00637

Li, F.-W., Villarreal, J. C., Kelly, S., Rothfels, C. J., Melkonian, M., Frangedakis, E., et al. (2014). Horizontal transfer of an adaptive chimeric photoreceptor from bryophytes to ferns. Proc. Natl. Acad. Sci. U.S.A. 111, 6672-6677. doi: 10.1073/pnas.1319929111

Luesse, D. R., DeBlasio, S. L., and Hangarter, R. P. (2006). Plastid movement impaired 2, a new gene involved in normal blue-light-induced chloroplast movements in Arabidopsis. Plant Physiol. 141, 1328-1337. doi: 10.1104/pp.106.080333

Maekawa, T., Tsutsui, I., and Nagai, R. (1986). Light-regulated translocation of cytoplasm in green alga Dichotomosiphon. Plant Cell Physiol. 27, 837-851.

Mineyuki, Y., Kataoka, H., Masuda, Y., and Nagai, R. (1995). Dynamic changes in the actin cytoskeleton during the high-fluence rate response of the Mougeotia chloroplast. Protoplasma 185, 222-229. doi: 10.1007/BF0 1272863

Mizukami, M., and Wada, S. (1981). Action spectrum for light-induced chloroplast accumulation in a marine coenocytic green alga, Bryopsis plumosa. Plant Cell Physiol. 22, 1245-1255.

Navrot, N., Gelhaye, E., Jacquot, J.-P., and Rouhier, N. (2006). Identification of a new family of plant proteins loosely related to glutaredoxins with four CxxC motives. Photosynth. Res. 89, 71-79. doi: 10.1007/s11120-0069083-7

Oikawa, K., Kasahara, M., Kiyosue, T., Kagawa, T., Suetsugu, N., Takahashi, F., et al. (2003). CHLOROPLAST UNUSUAL POSITIONING1 is essential for proper chloroplast positioning. Plant Cell 15, 2805-2815. doi: 10.1105/tpc. 016428

Oikawa, K., Yamasato, A., Kong, S.-G., Kasahara, M., Nakai, M., Takahashi, F., et al. (2008). Chloroplast outer envelope protein CHUP1 is essential for chloroplast anchorage to the plasma membrane and chloroplast movement. Plant Physiol. 148, 829-842. doi: 10.1104/pp.108. 123075

Sakai, T., Kagawa, T., Kasahara, M., Swartz, T. E., Christie, J. M., Briggs, W. R., et al. (2001). Arabidopsis nph1 and npl1: blue light receptors that mediate both phototropism and chloroplast relocation. Proc. Natl. Acad. Sci. U.S.A. 98, 6969-6974. doi: 10.1073/pnas.101137598

Sato, Y., Wada, M., and Kadota, A. (2001). Choice of tracks, microtubules and/or actin filaments for chloroplast photo-movement is differentially controlled by phytochrome and a blue light receptor. J. Cell Sci. 114, 269-279.

Schmidt von Braun, S., and Schleiff, E. (2008). The chloroplast outer membrane protein CHUP1 interacts with actin and profilin. Planta 227, 1151-1159. doi: 10.1007/s00425-007-0688-7

Senn, G. (1908). Die Gestalts- und Lageveränderung der Pflanzen-Chromatophoren. Stuttgart: Engelmann.

Shen, Z., Liu, Y.-C., Bibeau, J. P., Lemoi, K. P., Tüzel, E., and Vidali, L. (2015). The kinesin-like proteins, $\mathrm{KAC1} / 2$, regulate actin dynamics underlying chloroplast light-avoidance in Physcomitrella patens. J. Integr. Plant Biol. 57, 106-119. doi: 10.1111/jipb.12303

Suetsugu, N., Higa, T., Kong, S.-G., and Wada, M. (2015). PLASTID MOVEMENT IMPAIRED1 and PLASTID MOVEMENT IMPAIRED1-RELATED1 mediate photorelocation movements of both chloroplasts and nuclei. Plant Physiol. 169, 1155-1167. doi: 10.1104/pp.15.00214

Suetsugu, N., Kagawa, T., and Wada, M. (2005). An auxilin-like J-domain protein, JAC1, regulates phototropin-mediated chloroplast movement in Arabidopsis. Plant Physiol. 139, 151-162. doi: 10.1104/pp.105. 067371

Suetsugu, N., Sato, Y., Tsuboi, H., Kasahara, M., Imaizumi, T., Kagawa, T., et al. (2012). The KAC family of kinesin-like proteins is essential for the association of chloroplasts with the plasma membrane in land plants. Plant Cell Physiol. 53, 1854-1865. doi: 10.1093/pcp/ pcs 133

Suetsugu, N., and Wada, M. (2007). Chloroplast photorelocation movement mediated by phototropin family proteins in green plants. Biol. Chem. 388, 927-935. doi: 10.1515/BC.2007.118

Suetsugu, N., Yamada, N., Kagawa, T., Yonekura, H., Uyeda, T. Q. P., Kadota, A., et al. (2010). Two kinesin-like proteins mediate actin-based chloroplast 
movement in Arabidopsis thaliana. Proc. Natl. Acad. Sci. U.S.A. 107, 8860-8865. doi: 10.1073/pnas.0912773107

Tsuboi, H., and Wada, M. (2012). Distribution pattern changes of actin filaments during chloroplast movement in Adiantum capillus-veneris. J. Plant Res. 125, 417-428. doi: 10.1007/s10265-011-0444-8

Usami, H., Maeda, T., Fujii, Y., Oikawa, K., Takahashi, F., Kagawa, T., et al. (2012). CHUP1 mediates actin-based light-induced chloroplast avoidance movement in the moss Physcomitrella patens. Planta 236, 1889-1897. doi: 10.1007/s00425012-1735-6

Wada, M., and Suetsugu, N. (2013). "Chloroplast motility," in The Plant SciencesCell Biology, eds S. M. Assmann and B. Liu (Berlin, DE: Springer-Verlag), $1-16$.

Wagner, G., Haupt, W., and Laux, A. (1972). Reversible inhibition of chloroplast movement by cytochalasin B in the green alga Mougeotia. Science 176, 808-809. doi: 10.1126/science.176.4036.808

Whippo, C. W., Khurana, P., Davis, P. A., DeBlasio, S. L., DeSloover, D., Staiger, C. J., et al. (2011). THRUMIN1 is a light-regulated actin-bundling protein involved in chloroplast motility. Curr. Biol. 21, 59-64. doi: 10.1016/j.cub.2010.11.059

Yamashita, H., Sato, Y., Kanegae, T., Kagawa, T., Wada, M., and Kadota, A. (2011). Chloroplast actin filaments organize meshwork on the photorelocated chloroplasts in the moss Physcomitrella patens. Planta 233, 357-368. doi: 10.1007/00425-010-1299-2

Conflict of Interest Statement: The authors declare that the research was conducted in the absence of any commercial or financial relationships that could be construed as a potential conflict of interest.

Copyright (c) 2016 Suetsugu and Wada. This is an open-access article distributed under the terms of the Creative Commons Attribution License (CC BY). The use, distribution or reproduction in other forums is permitted, provided the original author(s) or licensor are credited and that the original publication in this journal is cited, in accordance with accepted academic practice. No use, distribution or reproduction is permitted which does not comply with these terms. 